Portland State University

PDXScholar

$5-24-2018$

\title{
Atypical Speech Development and Working Memory Recall Ability
}

Savannah LeeAnn Couch

Portland State University

Follow this and additional works at: https://pdxscholar.library.pdx.edu/honorstheses Let us know how access to this document benefits you.

\section{Recommended Citation}

Couch, Savannah LeeAnn, "Atypical Speech Development and Working Memory Recall Ability" (2018). University Honors Theses. Paper 565.

https://doi.org/10.15760/honors.572

This Thesis is brought to you for free and open access. It has been accepted for inclusion in University Honors Theses by an authorized administrator of PDXScholar. Please contact us if we can make this document more accessible: pdxscholar@pdx.edu. 


\section{Introduction}

\section{Speech Development and Working Memory}

Speech is a complex skill developed over time. Speech acquisition includes learning to discriminate auditory and perceptual information, understanding the meaning of sounds in a language, learning how to produce and use meaningful sounds and structures in words and sentences. As a child gains practice with speech, perceptual patterns of sounds are stored in memory for later retrieval by infants and toddlers in the developmental stage of speech acquisition.

Working memory is an important component of speaking. There are several approaches to looking at working memory. Some of the more widely accepted approaches include the Pascual-Leone model, the Anders Ericsson model and Nelson Cowan's model. However, the most widely considered models are the unitary model and the three component model.

The unitary model suggests short term memory is a concurrent storage and manipulation process. During this process tasks are devised to combine processing and storage (Baddeley 1992). Cognitive skills such as, reading, comprehension and reasoning are theorized to be contingent on the overall capacity of this unitary model. The unitary model is theorized to identify and store input simultaneously.

The second approach, the three component model, has focused mainly on the structure of working memory as a neuropsychological system. The three component model paradigm is complimentary to the unitary model in that it stresses the functional importance that working memory has in facilitating cognitive skills (Baddeley \& Hitch, 2000). However, it separates the 
verbal and visual-spatial components. This separation allows for exploration of the specific contribution of these subsystems to complex tasks (Baddeley 1992).

The aforementioned unitary approach has been considered "short-term memory" because short-term memory works as a unit to process stimuli and store that stimuli before decay. The three component approach was adopted by Alan Baddeley and Graham Hitch to emphasize the differences in their three component model and the earlier unitary model (Baddeley 2003). This thesis is set to explore a potential relationship in speech production and Baddeley and Hitch's three component model of working memory.

Alan Baddeley and Graham Hitch introduced their working memory model in the early 1970s aiming to explores possible neuropsychological subsystems that aid in the acquisition of native phonology. Baddeley and Hitch adopted their model from earlier models of short term memory but provided more detail in how working memory might play an important role in the ability to correctly decode and accurately produce native phonology. The role of working memory as a tool in speech production is currently debated by several research studies. This thesis aims to explore what we know about speech and language learning as well as working memory models and how those models effect speech production.

\section{Speech Sound Disorders and Working Memory}

Children in the developmental stages of speech and language acquisition rely heavily on skills in multiple domains such as, perception, cognition, motor, and linguistics. (Farquharson, Hogan, \& Bernthal, 2017). It can be said if there is a deficit in one or more of these domains a child will not develop speech and language typically. Within the field of speech-language pathology, delays in accurate production of developmentally appropriate speech sounds is 
identified as speech sound disorders (SSDs) (Lewis, Freebairn, Tag, Ciesla, Iyengar, Stein, \& Taylor, 2015).

The seminal work of Alan Baddeley and Graham Hitch suggest that people whose speech errors stem from the loss to assemble speech-motor plans do not show evidence of cognitive phonological rehearsal. Baddeley and Hitch present that although short-term working memory deficits are not majorly linked to comprehension deficits, they do show an overall effect on new phonological learning. This theory suggests that speech-motor planning is essential to rehearsal, consequently an important process for phonological output (Baddeley \& Hitch, 1994).

Complimentary to Baddeley and Hitch's 1994 publication, Anne-Marie Adams and Susan Gathercole designed a study looking at spontaneous speech output and phonological memory in 3-year-olds. They concluded that there was a significant difference across the sample. The children with strong phonological memory demonstrated language that was more grammatically complex and they had a richer lexical bank. Thus, a highly debated hypothesis is presented: that working memory has an impact on development in speech and language learning (Adams \& Gathercole, 1995).

Short-term working memory has been theorized as a vital subsystem to successful phonological acquisition. Alan Baddeley and colleagues suggest that "poor memory in children with SLI [specific language impairment] is consistent with the hypothesis that memory limitations are the root cause of the language impairment" (Baddeley, Papgno, \& Vallar, 1988). However, the opposite is possible as well, in that poor language in children may result in poor functional working memory (Baddeley, Papgno, \& Vallar, 1988). Thus it is a possibility that working memory ability can predict a child's ability to develop accurate phonology, thus resulting in impaired phonological output. These two conflicting ideas make it hard to determine 
the cause and effect relationship between typical speech production and short term working memory ability. To understand the relationship between working memory and typical speech production, the relationship between language learning and working memory must be explored.

The phonological loop has been credited to not only play a role in mediating the acquisition of syntactic knowledge but also individual word learning. (Baddeley, Gathercole \& Papagno, 1998) To further explore this hypothesis, Baddeley co-conducted a study with Constanza Papagno, and Giuseppe Vallar (1988), exploring if someone with a working memory deficit could learn words from an unfamiliar foreign language. The researchers required a woman with a clear working memory deficit to learn eight items from Russian vocabulary. They explored her ability to learn the foreign words with her ability to learn associative pairs of words from her native language by comparing her scores in both foreign and native word learning with controlled subjects. The controlled subjects were able to learn the new foreign words over 10 trials, whereas the participant with memory deficits was not able to learn the foreign vocabulary words. Baddeley later suggests in a 2003 publication that, based on the findings from the aforementioned controlled trial, the phonological loop does aid in the ability to learn new words (Baddeley, 2003a).

Baddeley tests his hypothesis further by implementing the articulatory suppression phenomenon. This phenomenon is designed to impair the performance of the phonological loop by disrupting the articulatory subvocal rehearsal system. To test this hypothesis, 14 adults were asked to utter an irrelevant sound while learning foreign words. By uttering the irrelevant sound, the participants' articulatory control system was interrupted by requiring the participants to suppress the rehearsal phase of language acquisition. It was hypothesized that by disrupting the rehearsal system, the ability to learn a foreign word would be weakened. The findings suggest 
that the ability to learn a word from a foreign language was disrupted by the suppression of the rehearsal system. However the disruption did not impair the participants' ability to lean a word from their native language.

The research done by Baddeley suggests that the phonological loop works as a backup system for language comprehension. In the aforementioned study, it is discussed that auditory input must be refreshed at a deeper level, within the articulatory control system, before it can be stored in long-term memory (Baddeley et al., 1998). If a child in the developmental stages of speech learning is not able to refresh the phonemes of their native language within the articulatory control system consistently, their ability to recall and produce those phonemes will be impaired. Adams and Gathercole (1995) as well as Baddeley and Hitch (1994) highlight this hypothesis by agreeing that the short-term memory does play a role in speech and language learning.

\section{The Phonological Loop as a System}

There are several conceptualisations of working memory, all with the overarching idea that the working memory process is a "holding tank" (Farquharson et al., 2017). The holding tank idea is that the working memory process stores information until it is either transferred to long term working memory or forgotten. Baddeley and Hitch published a working memory model that incorporates three sub systems of working memory: the phonological loop, the visuospatial sketch pad, and the central executive. Later in research, the episodic buffer was added as the fourth component. Their model is used to understand the relationship between working memory and the process of phonological acquisition, storage, and recall. The phonological loop is broken down into two sub components, the phonological store, and articulately control system. The phonological store is a storage system that temporarily holds verbal information. To avoid 
rapid decay of the stored information, the second component, the articulatory control system, refreshes the phonological store via the subvocalization process (Baddeley, 2003b). Figure 1 explains how the central executive works as a navigation system for the phonological loop allocating how information is going to be manipulated for short term storage or long term retrieval.

\section{Working Memory Model (Baddeley and Hitch, 1974)}

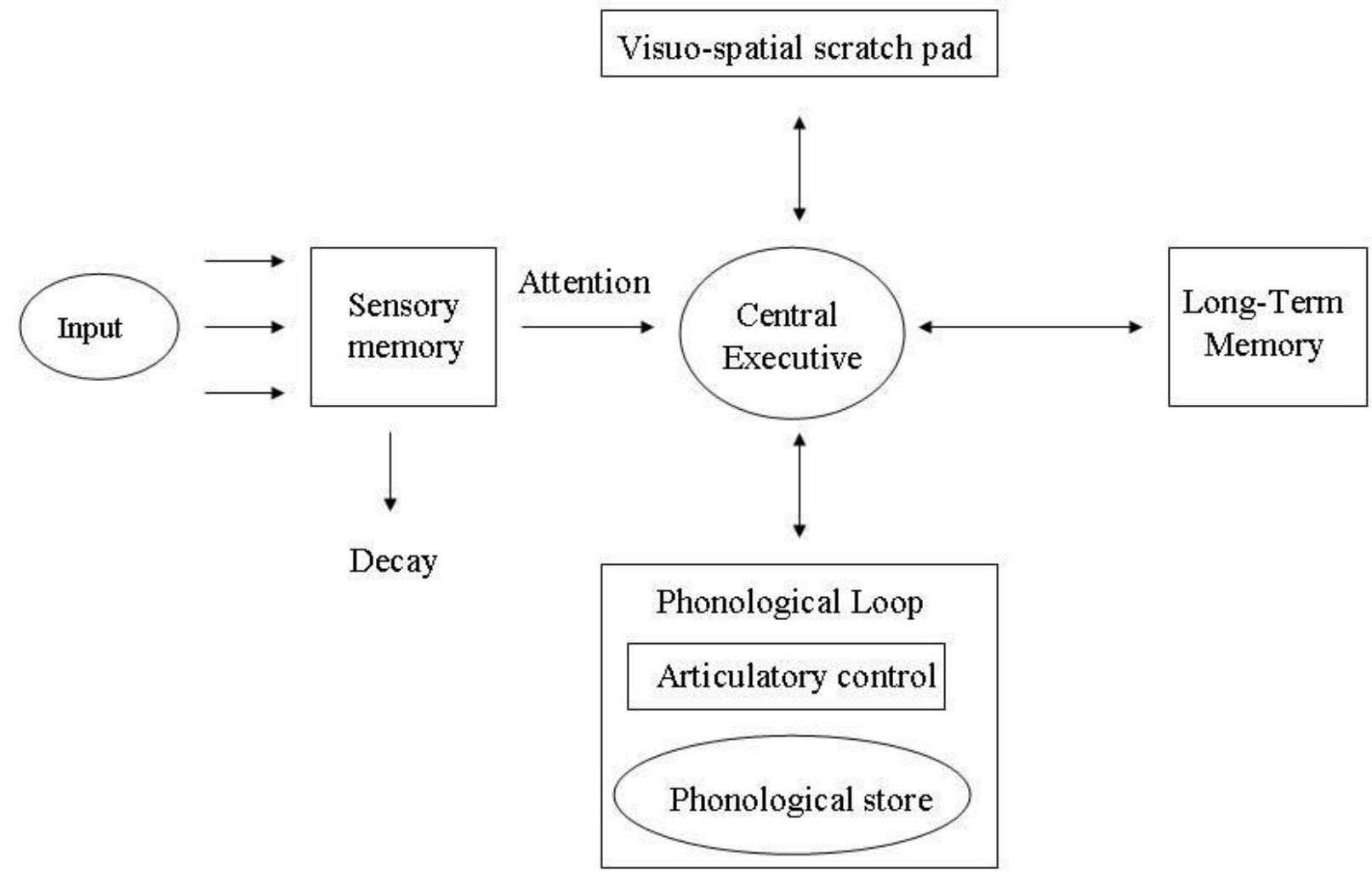

(from McLeod, 2008)

Figure 1 The central executive component directs information to be manipulated and stored. The information is sorted based on stimuli type. The visuo-spatial sketch pad stores symbolic information or pictures. The phonological loop houses the articulatory control system which refreshes speech and language input. All of these components work together to store input before rapid decay. 
The phonological store houses the unit for auditory information processing such as speech. Within the phonological loop, the phonological store processes incoming information and organizes it based in similarity. The phonological store serves as a limited capacity space that allows the temporarily stored information to be refreshed or decay. The second system within the phonological loop is that articulatory control mechanism. This mechanism serves two functions for the phonological loop: it stores verbal input, and codes orthographic information into phonological information. Both functions aid in later manipulation and long term memory storage. Baddeley explains, subvocal articulation is the process that refreshes information within the phonological loop to avoid rapid decay (Baddeley, 2007). The need for this subvocal component suggest that children in the developmental stages of language learning and speech acquisition who have atypical speech production skills could also have impairment in accurately reactivating phonological information before it decays in memory. (Farquharson et al., 2017).

The articulatory control system is a sub-articulatory rehearsal process that has two primary functions. When verbal material is presented as a stimulus it is directly sent to the phonological store for later manipulation. When visual/orthographic stimuli are presented, the rehearsal process takes over to recode the stimuli into phonologic information (Silveri, DiBetta, Filippini, Leggio \& Molinari, 1998). The first function of the articulatory control system is to recirculate phonological information before it decays. The second function is to recode orthographic stimuli into phonological information that can be stored and later retrieved (Silveri et al., 1998).

In addition to the phonological loop and central executive, the two other components of Baddeley and Hitch's working memory model are the visual-spatial sketch pad and the episodic buffer. The visuo-spatial sketch pad is described as the unit that stores visual information, such as pictures or symbols. The function of the central executive is to assign information being 
processed to either the the phonological loop or the visual-spatial sketch pad component. These two components work in concert to determine information that is spoken and information that is visually represented.

The episodic buffer is assumed to be the fourth component of Baddeley and Hitch's working memory model. It is a limited capacity storage system that integrates information from different sources temporarily. It is controlled by the central executive which relays information from visual-spatial and verbal stimuli where it is then manipulated or modified (Baddeley, 2000). The buffer acts as a temporary interface system between the short-term working memory and long term memory storage, binding information into coherent episodes. This is an important process in working memory and cognitive functioning because these episodes are assumed to be retrievable from long term memory storage.

The phonological loop system as a whole incorporates the central executive to allocate spoken language to the phonological store where it is either learned or forgotten. Within the phonological store the articulatory rehearsal mechanism refreshes the information through subvocal rehearsal before the information decays (Farquharson et al., 2017). This system suggests that the articulatory rehearsal mechanisms for children with speech sound disorders will be negatively impacted by their speech production challenges. (Farquharson at al., 2017).

\section{Phonological Working Memory Tasks}

The phonological store is theorized to be associated with two phenomena regarding working memory recall. These two phenomena are the phonological similarity effect and the word length effect. These phenomena occur when increasing the length of a word in a set or increasing the phonological similarity of word in a set negatively affects recall performance. 
Ashley Couture and Rebecca McCauley (2000) examined specific language impairment and the effect on cognitive working memory using the phonological similarity effect and the word length effect, predicting that children with impaired phonology will score more poorly on working memory tasks than their typically developing age-matched peers .

Couture and McCauley recruited 10 children between the ages of 5;11 and 7;2. Five of the participants had phonological impairment and the other five were age matched peers. The study addressed the word length effect and the phonological similarity effect in their study using two phonological working memory tasks. During the word length effect task participants were asked to recall sets of one syllable and three syllable word sets. During the phonological similarity task, the participants were asked to recall sets of phonologically similar words and phonologically dissimilar words. The hypothesis of this study was based on the findings that children with phonological impairment will demonstrate difficulties with working memory tasks. The results of this study show that the participants with impaired phonology did perform more poorly than their agedmatched peers, but their impaired phonology is not the cause of their overall performance. Couture and McCauley presented the idea that children with phonological impairment, are likely experiencing inefficient phonological working memory. Therefore they are expected to have sensitivity to the word length and phonological similarity (Couture, McCauley 2000). Couture and McCauley suggest that it is not the short term memory store but their long term memory abilities that explains the deficits in recall ability in children with impaired phonology. Thus the outcome of this study suggests that the poorer overall recall performance by the group of participants who had phonological impairments was not due to the specific mechanism in the phonological loop as modeled by Baddeley and Hitch. Couture and McCauley state that more research is warranted in this area. 


\section{Working Memory in School-aged Children}

Many of the studies done have been centered on school-aged children. It can be said that these children have already developed the phonology in their native languages. Farquharson, Hogan, and Bernthal (2017) explored school-aged children's ability to repeat back four nonwords in serial order. The participants in their study were 40 school-aged children between the ages of 7;5 and 11;8. Half of the participants were documented to have speech sound disorders and the other half were typically developing. The participants were divided into groups based on speech level. Farquharson used the Goldman-Fristoe Articulation Assessment version 2 (GFTA2) to assess the participants speech and designed the phonological loop tasks to examine serial recall (the ability to repeat a string of phonologic information in the same order that it was presented). It was hypothesized that the children with SSDs would perform more poorly on the phonological loop tasks than their age-matched peers. The results of this study showed poor phonological working memory for participants with SSDs. However, the data speak to a more significant relationship between nonverbal intelligence and phonological working memory. Farquharson discusses the need for more research looking at the complex relationship between poor phonological working memory and linguistic knowledge linked to children with SSDs.

\section{The Current Study}

The current research widely supports Baddeley and Hitch's three component model, with the addition of the episodic buffer in the phonological loop subsystem playing a key role in the ability to store auditory and visual stimuli. The phonological loop uses two mechanisms, the articulatory control system and the phonological store to refresh stimuli before it decays. Research supports this theory however, there are several questions regrading the relationship 
between Baddeley and Hitch's model and speech production ability. This research study explored the role adequate working memory has on the developmental stages of speech.

Using Baddeley and Hitch's three component working memory model, we explored whether deficits in speech-motor planning affect the rehearsal process within the limits of the phonological loop arena by comparing children with speech sound errors to their typically developing peers. This research is intended to further develop our collective understanding of the possible breakdown that occurs between working memory and speech production in typical and atypical developing pre- to school-aged kids. In particular, we explored whether children with speech sound errors will also have deficits in the rehearsal process of working memory and will their working memory recall ability reflect that? This question allows us to research early stages of speech production and discuss how a speech sound disorder may affect children's working memory scores based on their ability to subvocalize phonemes, consequently producing incorrect speech. If the two are connected, it would suggest that working memory aids in the process of recalling and using correct phonology.

\section{Methods}

\section{Participants:}

Forty-one children between the ages of 5;0-5;11 and 8;0-8;11 were recruited for this study. All participants were recruited from the Portland metro area through PSU student listservs, fliers distributed within Portland Public Schools, and reaching out to parents in the Portland metro community through acquaintances and community listservs such as nextdoor.com. To compensate for the low number of participants presenting with speech- 
language errors, additional recruitment was undertaken through Portland State University Speech and Hearing faculty members' clients and families.

Of the 41original children, seven of the participants were excluded from this study due to unfinished screening data, failure to pass a hearing screening, or because they were not monolingual English speakers. Participant exclusion data is shown in Figure 1 Thirty-three participants met total inclusion criteria. Twenty-two of the participants included demonstrated typical speech and language development.

The participants sampled for this study were grouped into two categories: those with speech-language deficits, the atypical group (AG). Test group participant data is shown in Table 1. The second group was the participants who presented with typical speech-language development. The participants included in the control group (CG) are age matched peers to the test group. Control group participant data is shown in Table 2. Of the 34 participants included in this study 12 presented with speech-language deficits, including atypical phonology, sensory processing deficits or other motor planning challenges reported by parent/legal guardian and/or graduate student project manager. For the purpose of this study we focused on speech scores, determining the test group based on scores from a standardized articulation test.

The atypical group included 12 participants split into two groups based on age. Six of the participants in the atypical group were within the $5 ; 0-5 ; 11$ age group and six were in the $8 ; 0-8 ; 11$ age group. The control group included 22 age matched peers with typical speech and language used for specific comparison. Of the 22 participants in the CG 10 were in the 5;0-5;11 age group and 12 participants were in the 8;0-8;11 age group. The participants were grouped by age for developmental comparison and then were separated further by recorded speech and language 
behaviors. The mean age of the participants in the 5;0-5;11 group was 5;3. The mean age of the participants in the $8 ; 0-8 ; 11$ group was $8 ; 3$. 


\section{Table 1}

\begin{tabular}{|c|c|c|c|c|c|}
\hline Participant \# & Age & Gender & Ethnicity & Noted History & Speech Level \\
\hline 9 & $5 ; 6$ & $\mathrm{~F}$ & Asian & None & Atypical \\
\hline 23 & $5 ; 2$ & $\mathrm{~F}$ & White & Parent report anxiety/ sensory processing issues & Atypical \\
\hline 26 & $5 ; 10$ & M & White/Asian & Born at 32 weeks/severe feeding disorder & Atypical \\
\hline 5 & $5 ; 9$ & $\mathrm{~F}$ & White & Late talker & Atypical \\
\hline 36 & $5 ; 2$ & $\mathrm{~F}$ & White & Fronted severely & Atypical \\
\hline 41 & $5 ; 2$ & M & White & None & Atypical \\
\hline 6 & $8 ; 10$ & M & White & Late talker/articulatory challenges & Atypical \\
\hline 13 & $8 ; 8$ & M & White/Asian & Speech assessment indicative of SSD & Atypical \\
\hline 28 & $8 ; 3$ & M & White/Asian & Trouble with "th" & Atypical \\
\hline 24 & $8 ; 10$ & M & White & None & Atypical \\
\hline 33 & $8 ; 7$ & M & White & None & Atypical \\
\hline 39 & $8 ; 2$ & $\mathrm{~F}$ & White & None & Atypical \\
\hline
\end{tabular}




\section{Table 2}

\begin{tabular}{|c|c|c|c|c|c|}
\hline Participant \# & Age & Gender & Ethnicity & Noted History & Speech Level \\
\hline 12 & $5 ; 9$ & $\mathrm{~F}$ & White/Asian & None & Typical \\
\hline 20 & $5 ; 3$ & M & White & Referred to NNRES & Typical \\
\hline 29 & $5 ; 6$ & $\mathrm{~F}$ & African American & None & Typical \\
\hline 14 & $5 ; 10$ & M & American Indian & None & Typical \\
\hline 4 & $5 ; 10$ & M & White & None & Typical \\
\hline 7 & $5 ; 1$ & $\mathrm{~F}$ & White & None & Typical \\
\hline 11 & $5 ; 1$ & $\mathrm{~F}$ & White & None & Typical \\
\hline 17 & $5 ; 1$ & $\mathrm{~F}$ & White & None & Typical \\
\hline 30 & $5 ; 0$ & M & White & None & Typical \\
\hline 37 & $5 ; 9$ & M & White & None & Typical \\
\hline 32 & $8 ; 9$ & M & White & None & Typical \\
\hline 18 & $8 ; 8$ & M & White & None & Typical \\
\hline 31 & $8 ; 9$ & M & White & None & Typical \\
\hline 25 & $8 ; 0$ & $\mathrm{~F}$ & White & None & Typical \\
\hline 1 & $8 ; 11$ & M & White & Assessment for stuttering later & Typical \\
\hline & & & & reported not a PWS & \\
\hline 2 & $8 ; 5$ & $\mathrm{~F}$ & White & None & Typical \\
\hline 10 & $8 ; 11$ & M & White & None & Typical \\
\hline 15 & $8 ; 2$ & M & Asian/White & None & Typical \\
\hline 21 & $8 ; 2$ & $\mathrm{~F}$ & White & None & Typical \\
\hline 22 & $8 ; 11$ & $\mathrm{~F}$ & White & None & Typical \\
\hline 27 & $8 ; 1$ & $\mathrm{M}$ & White & None & Typical \\
\hline 38 & $8 ; 2$ & $\mathrm{~F}$ & White/Asian & None & Typical \\
\hline
\end{tabular}

\section{Procedures:}

The families of participants were welcomed into the research setting on the weekends or no school days for data collection. The evaluation for this study consisted of six different assessment tools. The Diagnostic Evaluation of Articulation and Phonology (DEAP), The 
Clinical Evaluation of Language Fundamentals (version 5) (CELF-5), two experimental cognitive working memory tasks, The School-age Language Assessment Measures (SLAM), and verb-noun sentence elicitations. The protocol takes between 1.5 to 3 hours from consent to compensation. Data regarding the participants' speech-language abilities and a short overview of medical history and as background information were collected from the parent/legal guardian. During this time, the examiner starts to build rapport with the participant. Upon consent from the participant's parent/legal guardian, the examiner confirms assent from the child and begins the assessment.

A hearing screening is also conducted by the research examiner or research assistant. The participants are tested within The American National Standards Institute (ANSI) hearing screening standards at $25 \mathrm{~dB}$ for $500 \mathrm{~Hz}, 1000 \mathrm{~Hz}, 2000 \mathrm{~Hz}$, and $4000 \mathrm{~Hz}$ using a calibrated portable audiometer. All participants are required to pass the hearing screening prior to all other assessment activities.

The next step is the administration of the experimental cognitive working memory tasks. The working memory tasks included are a Competing Language Task and a Counting Span Task. The Counting Span task is presented as a booklet style assessment. The participant is asked to physically point to stars and count them aloud. When the participant finishes, they must repeat back the number of stars on each page in the order that they counted them. The order is an important step for scoring because a child could repeat back a series of numbers, and by chance get them correct. For example, if the sequence of numbers is $6,3,5,4,2$, and the participant responds with $1,2,3,4,5$, there would be a total of four correct responses, this would tell us little about the child's functioning working memory. The sections grow in length every three elicitations and the participant repeated back six numbers by the end of the task. 
The second working memory task was a Competing Language Task. The participant was read a true or false sentence out loud by the tester. The participant answered true/false or yes/no after the sentence had been said. Then they were asked to remember and repeat the last word of the sentence. The child was asked to remember up to six words by the end of the assessment, however the order, unlike the counting task, was not important. The order is not specifically important for this task because it is far less likely that the participants will be able to produce random words and score correctly.

The DEAP is the next assessment included in the screening to collect data on each participant's phonological development and to measure their ability to produce American English phonemes. The DEAP is applicable for children ages 5-8. It targets single words, broken into sections by syllable initial (consonant/consonant clusters), vowel, and syllable final (consonant/consonant clusters).

The next step in the screening is the introduction of the verb-noun sentence elicitation. The procedure for the next portion is as follows: the child sits across from the tester at a small table. The examiner reads a short subject-verb-object (SVO) sentence and counts to 5, the participant then repeats back the same sentence as naturally as possible, while looking into the camera. The counting ensures the expungement of sensory memory. This task is repeated multiple times throughout the screening for later data analysis purposes.

The CELF-5 is a comprehensive battery administered to assess the participant's language comprehension and extension. It is administered in sections throughout the assessment inbetween sentence elicitations. For this study, four sections of the CELF-5 were administered, Sentence Comprehension, Formulated Sentences, Word Structure and Recalling Sentences. The CELF-5 quantified the participant's developmental stage in language fundamentals. 
The SLAM cards "Dog Comes Home", and "Bunny Goes to School" are included to "elicit a language sample that can be analyzed in the context of typical language development, as well as the child's background (e.g., educational experiences, family, linguistic, and cultural background etc...)" (Crowley 2017, para. 1). The participant is asked several questions about the cards and they are asked to tell the whole story using the pictures on each card.

Once all the tasks are completed the child is given a prize for their work and they are compensated for their time.

\section{Equipment:}

Specialized recording equipment was used for later data retrieval and for scoring accuracy. The camera was set up to visually record each participant is a Panasonic P2HD during elicitations and the SLAM language sample assessment. The participants wear a headband that supported a Shure ULX S4 microphone during the video recording. In addition to the camera and microphone, Genaray Spectro LED spot lights are used to enhance the image and clarity of the visually recorded portions. The participant's voice is audio recorded throughout the entire assessment using a Tascam DR-05 recording device. This equipment is utilized to ensure accurate data retrieval for later scoring and analyzation as well as increasing the similarity across assessments. 


\section{Data Analysis}

This study compared the DEAP, experimental working memory tasks, and CELF-5 scores. We assessed each participants speech using the articulation subsection of the DEAP. Atypical errors are errors made by fewer than $90 \%$ of the normative sample at any age between $3 ; 0$ and 6;11. Consequently, there is no criterion number of times an atypical error pattern occurs for a child to be credited with its use. Some atypical errors patterns affect many syllables / words (e.g., word initial consonants deleted, all word final consonant delete except nasals, all consonant clusters marked by a bilabial fricative). Others occur to very restricted phonetic contexts (e.g., word final /1/ deletes after a back vowel)." (Dodd, 2002) Clinical judgment is used to determine atypical errors in real time. For both age groups, atypical errors were determined as types of errors made by fewer than $90 \%$ of the normative sample (Dodd, 2002). Adjusted scores are documented as 1-12. If a participant scored below a 6 they were placed in the test group, the DEAP standard score indicates if a participant has typical/atypical speech development. The mean and standard deviation scores are recorded and compared for both age groups. As mentioned there will be a discrepancy in the DEAP scores between CG and AG because the groups were defined by developmental speech production.

The CELF-5 was used to determine the participants' developmental language level. All participants in both CG and AG demonstrated high language scores. The CELF-5 is scored on a scale. Raw scores are taken for each subsection of the CELF-5 and converted into scaled scores. Each participant is then placed into a percentile group. The participants for this study all demonstrated extremely high language levels in both groups. By looking at the CELF-5 it can be 
determined if speech errors are co-occurring with language impairments. It can be determined that for this group of participants language deficits, in either group, are not a co-occurring factor.

The experimental cognitive working memory scores from the AG and the $\mathrm{CG}$ were compared in Excel using a point system. The Counting Span Task is worth 54 points based on the 54 opportunities for the participant to produce the correct number. The Competing Language Task is worth a total of 42 points based on the 42 opportunities for the participant to produce the correct word. Each of these tasks included two practice opportunities which are not included in the overall score. The DEAP and the CELF-5 were scored by the research assistant and examiner. The raw and scaled score data are recorded.

The SLAM language sample is included in the screening, the SLAM is video recorded however, no formal data is taken after the assessment. The SLAM is used to assess a participants' language level, semantics, pragmatics, syntax, and other language skills.

Sentence elicitations are repeated several times over the course of the assessment process. The elicitations are video recorded for later prosodic development data. For the purpose of this study, data from the SLAM and the sentence elicitations are not included.

\section{Results}

This study examined DEAP, CELF-5 and experimental working memory tasks to explorea potential relationship between working memory recall ability and speech development. It was hypothesized that the participants with atypical speech production, indicative of speech sound disorder, would also perform more poorly on the experimental working memory tasks. Both groups' scores were collected and recorded to compare speech production and language 
acquisition to their individual working memory task scores. Data are presented by age group below.

\section{5;0-5;11 Group}

The averages of 10 participants in the 5;0-5;11 age group who met the criteria for typical speech and language were calculated for each cognitive working memory task to determine normalcy across the sample. This group includes all participants from the CG. All participants included in this group were reported to have normal scores on the DEAP and the CELF-5. These typically-developing data are shown in Table 3.

The total average across these 10 participants whose speech was within functional limits (WFL) for the Counting Span Task (CST) was $11.7(A V G=11.7)$ with $S D=5.65$. The total average score for the Competing Language Task (CLT) across the 10 participants whose speech met the criteria for WFL inclusion was $16.6(A V G=16.6)$ with $S D=5.27$. The maximum correctly recalled numbers was 16 with minimum score of 1 . The maximum number of correctly recalled words for the CG on the CLT was 22 with a minimum score of 9. The data represented for the typically developing participants are shown in figure 2.0. For the participants in the AG group the CST $A V G=14$ with $S D=6.35$. The CLT $A V G=12.66$ and $S D=3.72$. The maximum correctly recalled numbers was 22 with the minimum of 6 . The data represented for the atypically developing participants are shown in Table 4.

The DEAP scores were recorded for the AG and CG. The DEAP scores are representative of typical speech development under standardized measures. The DEAP scores of the participants in the CG are used for this portion of the analysis. The 10 participants in the CG 
scored an $A V G=10.5$ with $S D=1.58$. The $A G$ scored an $A V G=5.66$ with $S D=1.63$. There is a significant difference in the scores of the AG and CG. However, we knew that when looking at this data there would be a difference considering the criteria for group inclusion was how the participant scored on the DEAP. The lower scores were indicative of speech challenges that could potentially affect the working memory recall ability.

The CELF-5 was administered and data was collected for the standardized scores of each participant. The CELF-5 reflects the developmental language stage each participant is in. The 10 participants included in the $C G$ had scored an $A V G=116.7$ and $S D=7.68$. The 6 participants included in the AG scored an $A V G=116.83$ and $S D=14.68$. As hypothesized the CELF-5 scores would not necessarily be lower in the AG. Because as we know, speech disorders do not always co-occur with language disorders.

\section{Table 3}

\section{Typical Group $(N=10)$}

Age 5;0-5;11

\begin{tabular}{|l|l|l|l|l} 
Participant \# & DEAP & CST & CLT & CELF-5 \\
\hline 4 & 10 & 15 & 10 & 118 \\
\hline 7 & 11 & 12 & 15 & 117 \\
\hline 11 & 11 & 5 & 22 & 125 \\
\hline 12 & 12 & 16 & 22 & 125 \\
\hline 14 & 12 & 9 & 17 & 102 \\
\hline 17 & 11 & 1 & 10 & 122 \\
\hline 20 & 10 & 10 & 21 & 109 \\
\hline 29 & 9 & 13 & 21 & 118 \\
\hline 30 & 7 & 16 & 9 & 122 \\
\hline 37 & 12 & 20 & 19 & 109 \\
\hline & & & & \\
\hline Average & 10.5 & 11.7 & 16.6 & 116.7 \\
\hline Standard Deviation & 1.58 & 5.65 & 5.27 & 7.68 \\
\hline Maximum & 12 & 16 & 22 & 125 \\
\hline Minimum & 7 & 1 & 9 & 102
\end{tabular}

Figure 2.0. The data are shown for the control group, age 5;0-5;11, by participant number and individual scores. The averages, standard deviations, maximum and minimum recalled words and numbers are displayed across the sample. 


\section{Table 4}

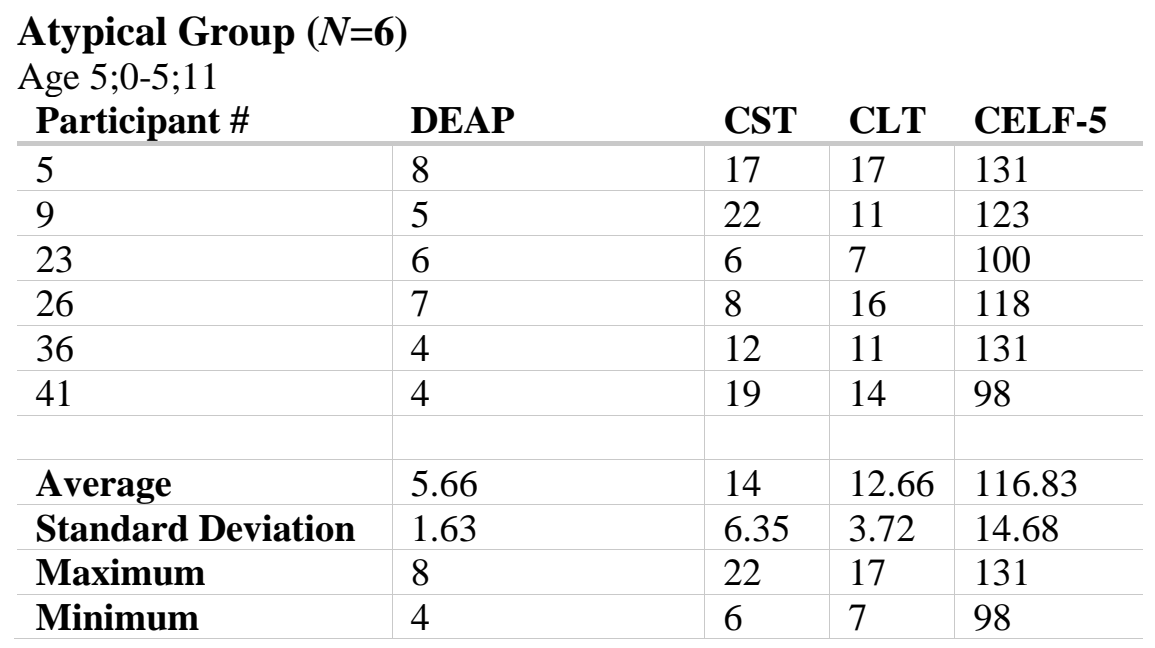

Figure 2.1. The data are shown for the atypical group, age 5;0-5;11, by participant number and individual scores. The averages, standard deviations, maximum and minimum recalled words and numbers are displayed across the sample.

\section{8;0-8;11 Group}

The averages of 12 participants in the 8;0-8;11 age group who met the criteria for typical speech and language were calculated for the cognitive working memory task activities to determine normalcy across the sample. All participants in this overall group were documented to have speech and language withing normal limits. These data are shown in Table 5.

The total average for the 12 participants on the CST, whose scores reflected speech and language WFL was $31.25(A V G=31.25)$ and $S D=5.27$. The total average for the 12 participants included in this group on the CLT was $25.83(A V G=25.83)$ and $S D=3.21$. The maximum correctly recalled numbers was 37 with a minimum score of 23 . The maximum correctly recalled words from the CLT for the CG was 34 with a minimum score of 23 . The data represented for 
the typically developing participants are shown in figure 2.3. For the 6 participants in the AG group the CST $A V G=14.5$ with $S D=6.92$. The CLT $A V G=12.16$ and $S D=3.71$. The 6 participants in the AG correctly recalled a maximum of 36 numbers, on the CSP with the minimum of 8 .The AG correctly recalled a maximum of 27 words on the CLT with a minimum of 13 . The data represented for the atypically developing participants are shown in Table 6.

The DEAP scores for both the AG and CG were documented as well. The DEAP scores are representative of typical speech development under standardized measures. The DEAP scores of the 12 participants in the CG are used for this portion of the analysis. The 12 participants in the $C G$ scored an $A V G=11.66$ and $S D=1.15$. It is important to note that the participants chosen for this group were chosen based on their high DEAP scores. Only participants who had DEAP scores reflecting high phonological development were included in the final CG group. While this does subject this study to some internal validity threats, it was essential to chose a control group based on their typically developing speech production. The AG group scored an $A V G=2.83$ with a $S D=2.40$. The participants selected for the AG were documented to have lower than average scores on the DEAP reflecting atypical speech development. As mentioned these groups were not randomized, they were selected to determine if there are discrepancies in the cognitive working memory scores and their DEAP scores.

The CELF-5 was administered and data was collected for the standardized scores of each participant. The CELF-5 reflects the developmental language stage each participant is in. The 12 participants included in the CG scored an $A V G=119.33$ and $S D=7.78$. The 6 participants included in the AG scored an $A V G=119.33$ and $S D=5.81$. There is no significant difference in the CELF-5 scores of the AG and CG for either age group. As noted in the 5;0-5;11 subsection, this is 
because speech disorders do not always co-occur with language disorders. It can be said that none of our participants demonstrated overt challenges with language in either age group.

\section{Table 5}

Typical Group $(N=12)$

Age 8;0-8;11

\begin{tabular}{|l|l|l|l|l} 
Participant \# & DEAP & CST & CLT & CELF-5 \\
\hline 1 & 12 & 24 & 24 & 113 \\
\hline 2 & 12 & 32 & 24 & 113 \\
\hline 10 & 12 & 34 & 28 & 113 \\
\hline 15 & 12 & 24 & 26 & 125 \\
\hline 18 & 12 & 23 & 24 & 115 \\
\hline 21 & 8 & 34 & 24 & 123 \\
\hline 22 & 12 & 28 & 28 & 129 \\
\hline 25 & 12 & 37 & 23 & 118 \\
\hline 27 & 12 & 37 & 23 & 118 \\
\hline 31 & 12 & 31 & 24 & 136 \\
\hline 32 & 12 & 37 & 34 & 120 \\
\hline 38 & 12 & 34 & 28 & 109 \\
\hline & & & & \\
\hline Average & 11.66 & 31.25 & 25.83 & 119.33 \\
\hline Standard Deviation & 1.15 & 5.27 & 3.21 & 7.78 \\
\hline Maximum & 12 & 37 & 34 & 136 \\
\hline Minimum & 8 & 23 & 23 & 113
\end{tabular}

The data are shown for the control group, age 8;0-8;11, by participant number and individual scores. The averages, standard deviations, maximum and minimum recalled words and numbers are displayed across the sample.

\section{Table 6}

Atypical Group $(N=6)$

\section{Age 8;0-8;11}

\begin{tabular}{|l|l|l|l|l}
\hline Participant \# & DEAP & CST & CLT & CELF-5 \\
\hline 6 & 4 & 35 & 27 & 118 \\
\hline 13 & 1 & 26 & 13 & 111 \\
\hline 24 & 7 & 8 & 16 & 118 \\
\hline 28 & 1 & 20 & 21 & 127 \\
\hline 33 & 1 & 36 & 26 & 125 \\
\hline 39 & 3 & 34 & 21 & 117 \\
\hline Average & & & & \\
\hline Standard Deviation & 2.83 & 26.5 & 20.66 & 119.33 \\
\hline Maximum & 2.40 & 10.98 & 5.46 & 5.81 \\
\hline & 7 & 36 & 27 & 127 \\
\hline
\end{tabular}




\begin{tabular}{l|l|l|l|l|} 
Minimum & 1 & 8 & 13 & 111
\end{tabular}

The data are shown for the atypical group, age 8;0-8;11, by participant number and individual scores. The averages, standard deviations, maximum and minimum recalled words and numbers are displayed across the sample.

\section{Discussion}

Baddeley and Hitch's working memory model suggests the rehearsal sub system in the phonological loop plays an important role in the ability to assemble speech motor plans (i.e., speech sounds) (Baddeley and Hitch, 2001). This hypothesis suggests that children who demonstrate challenges with speech production would also demonstrate challenges with working memory recall ability since the rehearsal system is directly linked to phonological output in the phonological loop component of the working memory model as explained by Baddeley and Hitch. As noted in the data analysis, the participants for this study were chosen directly based on their DEAP scores. The DEAP scores of the CG are significantly higher than the AG, but that is to be expected considering the design of this study.

The analysis of the participants' cognitive working memory task scores reflect some discrepancy across the AG and CG. In the 5;0-5;11 age group, the AG scored a higher average on the CST, which could be attributed to the larger sample size for the AG. There is a discrepancy in the CLT with the CG scoring a much higher average and smaller standard deviation. There is potentially a connection between phonological ability and the phonological sub vocal rehearsal system that is reflected by the scores in the AG and CG. The phonological sub vocal rehearsal system houses the sub system for orthographic information processing. This model would align appropriately with the notion that the participants who do not have speech WFL would also perform more poorly on a working memory task that does not allow them to 
rely on orthographic information. The CLT does not allow the participants to access any kind of visual stimulus or ask the tester any questions during the task. The CST does allow the participant to access a visual stimulus (stars and circles) which they are required to touch and count at the same time. Visually represented information is allocated by the central executive in the visual-spatial sketch pad component of the working memory system. Thus, the participants are accessing a different area of the working memory model. For children with SSDs, the phonological loop, where speech sounds are developed and learned, is the area that is hypothesized to be compromised by Baddeley and Hitch. Thus, the participants with atypical speech production would perform more poorly on tasks that do not allow them to access visual information.

The AG and CG in the 8;0-8;11 age group had more discrepancies than the $5 ; 0-5 ; 11$ age group. This could be attributed to a more specific scoring requirement and/or the overall phonological ability for 8 year olds. Kent (1976) has claimed that adult-like speech production will be achieved around the age of 11 years. It is hypothesized that the 8;0-8;11 year old age group would in fact have more discrepancies because there is more space for those discrepancies to present themselves. The participants in the 5;0-5;11 age group are going to have speech errors whether they are in the AG or CG based on developmentally appropriate speech errors.

\section{Conclusion}

The three component model for working memory suggests that children with SSDs will perform more poorly on tasks, including speech acquisition without the support of orthographic information. There are limits to this study and several questions remain for further research; 1) 
Could it be hypothesized that, given an image of the words the participants with SSDs were asked to recall after each sentence on the CLT, they would recall them with more accuracy? 2) Is it so that orthographic information is more easily stored and refreshed in the phonological loop system than speech?

The validity of this study is compromised by the sample of participants. All participants included demonstrated extremely high language levels as mentioned in data analysis. This could be attributed to the recruitment method used by research assistant. Many participants came from middle to high socioeconomic backgrounds, increasing the likelihood that parents/caregivers are using language that closely resembles assessment language.

Another possible limit to this study is the data collection method. Because data are coming from experimental procedures, (experimental cognitive working memory tasks) there is not a standardized score to compare the participants scores to. The scores that we are comparing are the averages from the $\mathrm{CG}$ (what we are considering normal) and the averages of the AG (what we are considering outside the range of normal, based on speech sound errors).

Lastly, there are limits to using The Diagnostic Evaluation of Articulation and Phonology standardized test. The DEAP is designed to test speech at the word level, it does not test connected speech. This test may not accurately reflect speech sound disorder in 5 and 8 year olds since single word productions may not reflect overall speech intelligibility at these ages. It is also normed so that if a child in a higher age range makes just one mistake they will drop to a much lower percentile than where they actually might be. It is recommended that future studies use a more comprehensive measure of speech to fully understand the effects of working memory on speech production. 


\section{References}

Adams, A.M., \& Gathercole, S. (1995). Phonological working memory and speech production in preschool children. Journal of Speech and Hearing Research, (38), 405-414. doi: 10.1044/jshr.3802.403

Baddeley, A. (1992). Working memory. Agriculture and Environmental Science. (225)5044, 556-570. Retrieved May 8, 2018, from http://stats.lib.pdx.edu/proxy.php?url=http://search.proquest.com.proxy.lib.pdx.edu/docview/213 $\underline{552179 \text { ? accountid }=13265}$

Baddeley, A. (2000). The episodic buffer: A new component in working memory? Trends in Cognitive Sciences, (4)11, 417-423. doi:https://doi-org.proxy.lib.pdx.edu/10.1016/S1364-6613(00)01538-2

Baddeley, A. (2003a). Working memory and language: An overview. Journal of Communication Disorders, (36), 189-208. Retrieved October 15, 2017.

Baddeley, A. (2003b). Working memory: Looking back and looking forward. Nature Reviews Nueroscience,(4)10, 829-839. Retrieved May 21, 2018.

Baddeley, A.D. (2007). Working memory, thought, and action. Oxford: Oxford University Press.

Baddeley, A., Gathercole, S., \& Papagno, C. (1998). The phonological loop as a language learning device. American Psychological Association, (105)1, 158-173. Retrieved May 5, 2018.

Baddeley, A., \& Hitch, G. (1994). Developments in the concept of working memory. Neuropsychology, (8)4, 485-493, Retrieved June 7, 2018.

Baddeley, A. D., Papagno, C., \& Vallar, G. (1988). When long-term learning depends on short-term storage. Journal of Memory and Language, (27), 586-595. Retrieved May 22, 2018.

Baddeley, A., \& Hitch, G. (2000). Development of working memory: Should the Pascual-Leone and the Baddeley and Hitch models Be merged? Journal of Experimental Child Psychology, (77)2, 128137. doi:https://doi-org.proxy.lib.pdx.edu/10.1006/jecp.2000.2592

Couture, A., McCauley, R., (2000). Phonological working memory in children with phonological impairment. Clinical Linguistics and Phonetics. (14)7, 499-517

Crowley, C. (2017, October 09). SLAM Dog Comes Home Cards. Retrieved February 27, 2018, from https://www.leadersproject.org/2015/03/18/slam-dog-comes-home-school-aged-languageassessment-measure/ 
Dodd, B. (2002). How to administer, score and interpret the 'Diagnostic Evaluation of Articulation and Phonology' (DEAP). Retrieved May 8, 2018, from

https://www.researchgate.net/profile/Barbara_Dodd/publication/43509881_Diagnostic_Evaluatio n_of_Articulation_and_Phonology_DEAP/links/576db62808ae10de6395d579/DiagnosticEvaluation-of-Articulation-and-Phonology-DEAP

Farquharson, K., Hogan, T.P., \& Bernthal, J. E. (2017). Working memory in school-age children with and without a persistent speech sound disorder. International Journal of Speech-Language Pathology, 1-12. Doi: 10.1080/1754907.2017.1293159

Lewis, B.A., Freebairn, L., Tag, J., Ciesla, A.A., Iyengar, S.K., Stein, C.M., \& Taylor, H.G. (2015). Adolescent outcomes of children with early speech sound disorders with and without language impairment. American Journal of Speech-Language, (24), 150-163. Doi:10.1044/2014_AJSLP$14-0075$

McLeod, S. (2008). [Image of working memory model] (2008), Retrieved from https://www.simplypsychology.org/working memory.html

Silveri, M. C., Di Betta, A. M., Filippini, V., Leggio, M. G., \& Molinari, M. (1998). Verbal short-term store-rehearsal system and the cerebellum Evidence from a patient with a right cerebellar lesion. Journal of Neurology, (121), 2175-2187. Retrieved April 8, 2018.

Thorn, A., \& Gathercole, S. (2001). Language differences in verbal short-term memory do not exclusively originate in the process of subvocal rehearsal. Psychonomic Bulletin and Review, (8)2, 357-364. Retrieved May 14, 2018. 